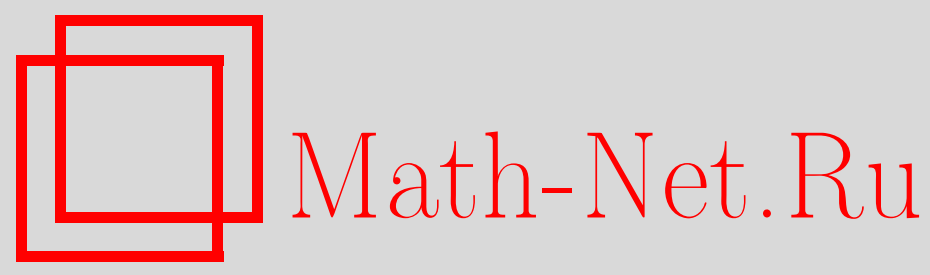

Н. Г. Парватов, Проблема выразимости в решетке с замыканием, Дискрет. матем., 2010, том 22, выпуск 4, 83-103

DOI: https://doi.org/10.4213/dm1121

Использование Общероссийского математического портала Math-Net.Ru подразумевает, что вы прочитали и согласны с пользовательским соглашением http://www.mathnet.ru/rus/agreement

Параметры загрузки:

IP: 3.81 .55 .215

26 апреля 2023 г., 13:40:11 
УДК 519.7

\title{
Проблема выразимости в решетке с замыканием
}

\author{
(ㄷ) 2010 г. $\quad$ Н. Г. Парватов
}

\begin{abstract}
В статье рассматривается проблема выразимости элемента полной решетки с операцией замыкания. Устанавливаются условия, при которых данная проблема имеет решение в виде конечной нижней окрестности. Отдельно рассматривается наиболее важный для приложения случай полной решетки $\mathscr{B}(P)$ упорядоченных включением подмножеств множества $P$. Показывается, что существование конечной нижней окрестности для каждого порожденного замкнутого элемента этой решетки влечет финитарность операции замыкания в ней. В случае решетки $\mathscr{B}(P)$ с финитарным замыканием устанавливаются конструктивные достаточные условия существования для ее элемента конечной нижней окрестности. Тем самым обобщается теорема А. В. Кузнецова о функциональной полноте. Отдельно рассматривается случай замыкания Галуа, индуцированного некоторым соответствием Галуа между решетками подмножеств. В этом случае устанавливаются необходимые и достаточные условия существования конечной нижней окрестности элемента решетки. Этим наряду с теоремой А. В. Кузнецова обобщается и теорема С. В. Яблонского о предикатно описываемых классах функций конечнозначной логики. Попутно с проблемами выразимости в статье рассматривается возможность задания замкнутых элементов решетки $\mathscr{B}(P)$, в частности, элементов нижних окрестностей, конечными запрещающими множествами при некотором предупорядочении множества $P$, а в случае замыкания Галуа - конечными описаниями. Доказанные теоремы сопровождаются примерами их использования.
\end{abstract}

\section{1. Проблема полноты и выразимости}

Пусть $\mathcal{M}$ - полная решетка (структура) (см. [1]). Иными словами, в множестве $\mathcal{M}$ задано отношение частичного порядка $\subseteq$ (называемое далее отношением включения) так, что для любой системы $\mathscr{A}$ элементов из $\mathcal{M}$ существует точная верхняя грань (объединение) $\bigcup \mathscr{A}$ и точная нижняя грань (пересечение) $\bigcap$ A. Пусть также в решетке $\mathcal{M}$ задана операция замыкания, обозначаемая '. Иными словами, в решетке $M$ для любого элемента $X$ определено его замыкание $X^{\prime}$ так, что $X \subseteq X^{\prime}, X^{\prime}=X^{\prime \prime}$ и $X^{\prime} \subseteq Y^{\prime}$ для любого элемента $Y$ из $M$, включающего $X$. Элементы, совпадающие со своими замыканиями, называются замкнутыми (элементами решетки $\mathcal{M}$ с замыканием '). Важным для дальнейшего примером решетки $\mathcal{M}$ является решетка $\mathscr{B}(P)$ всех подмножеств некоторого множества $P$, упорядоченных теоретико-множественным отношением включения. В этой решетке операции объединения $\cup$ и пересечения $\cap$ совпадают с одноименными теоретико-множественными операциями. Замыкание в решетке $\mathscr{B}(P)$ называется финитарным, если ее замкнутыми элементами являются в точности все подалгебры некоторой универсальной алгебры $[1,2]$, 
определенной на множестве $P$. Свойства операции замыкания ', равносильные финитарности, изучались в [3].

Как видно из сказанного, в нашем рассмотрении оказываются объекты разного уровня абстракции: элементы, множества и множества множеств. В связи с этим, чтобы избежать путаницы, договоримся множества элементов решетки $\mathcal{M}$ называть системами, а для их обозначения использовать каллиграфические заглавные буквы $\mathscr{A}, \mathscr{B}, \ldots$, в отличие от элементов решетки $\mathcal{M}$, для обозначения которых будут использоваться заглавные буквы $A, B, \ldots$ Это относится и к элементам решетки $\mathcal{M}=\mathscr{B}(P)$, для именования которых наряду со словом элемент дополнительно будем использовать слова множество и класс. Наконец, элементы множеств (классов) из $\mathscr{B}(P)$ будем обозначать малыми латинскими буквами $a, b, \ldots$

Введем в рассмотрение некоторые понятия. В решетке $\mathcal{M}$ с замыканием ' элемент $X$ станем называть $Y$-мажорирующим, если выполняется включение $X^{\prime} \supseteq Y^{\prime}$ (равносильное включению $X^{\prime} \supseteq Y$ ). Элемент $X$ станем называть $Y$-максимальным, если $X$ не является $Y$-мажорирующим в отличие от всех элементов $Z$ из $M$ таких, что $X \subset Z$. Если задана решетка $\mathcal{M}$ с замыканием, то систему всех ее $Y$-максимальных элементов будем обозначать $S(Y)$. Наконец, в решетке с замыканием элемент $X$ станем называть $Y$-порождающим, если выполняется равенство $X^{\prime}=Y^{\prime}$. При этом замкнутый класс $Y$ решетки $M=\mathscr{B}(P)$ с замыканием ', имеющий конечное $Y$-порождающее подмножество $X$ такое, что $X^{\prime}=Y$, называется конечно порожденным.

В дальнейшем нас будет интересовать проблема выразимости, а также проблема полноты элемента $Y$ в решетке $\mathcal{M}$ с замыканием '. Первая из этих проблем состоит в описании всех $Y$-мажорирующих элементов решетки $\mathcal{M}$. Вторая требует описания всех $Y$-порождающих элементов решетки $\mathcal{M}$ и совпадает с проблемой выразимости замкнутого элемента $Z=Y^{\prime}$ решетки

$$
M^{Z}=\{X \in M \mid X \subseteq Z\},
$$

в которой порядок и замыкание индуцированы из решетки $\mathcal{M}$. В некоторых случаях рассматривается также проблема $A$-полноты ( $A$-выразимости) элемента $Y$ в решетке $M$ с замыканием. Данная проблема для заданного элемента $A$ и заданного $A$-мажорирующего элемента $Y$ решетки $M$ с замыканием требует описания в ней всех $Y$-порождающих (соответственно $Y$-мажорирующих) и одновременно $A$-мажорирующих элементов. Она совпадет с проблемой полноты (соответственно вместимости) элемента $Y$ решетки

$$
\mathcal{M}_{A}=\{X \in \mathcal{M} \mid A \subseteq X\}
$$

с замыканием и порядком, индуцированными из $\mathcal{M}$, а также совпадает с проблемой полноты (выразимости) элемента $Y$ в решетке $\mathcal{M}$ с замыканием ${ }_{A}$, определенным как

$$
X_{A}^{\prime}=(X \cup A)^{\prime}
$$

для любого элемента $X$ из $\mathcal{M}$. В дальнейшем будем интересоваться в основном проблемой выразимости, которая, как видно из сказанного, является наиболее общей из перечисленных проблем.

В ряде случаев проблема выразимости допускает сведение к ряду формально более простых проблем выразимости. Несложно понять, например, что в полной решетке $M$ с замыканием проблема выразимости объединения $\bigcup \mathscr{A}$ системы $\mathscr{A} \subseteq \mathcal{M}$ сводится к ряду проблем выразимости элементов, принадлежащих системе $\mathscr{A}$. В частности, проблема выразимости множества $A$ в решетке $\mathscr{B}(P)$ сводится к ряду проблем выразимости одноэлементных множеств $\{a\}$ для всевозможных элементов $a$ из $A$. Несколько иначе в 
решетке $M$ с замыканием проблема выразимости объединения $A \cup B$ ее элементов $A$ и $B$ сводится к проблемам выразимости элементов $A$ и $A \cup B$ в соответствующих решетках $\mathcal{M}$ и $\mathcal{M}_{A}$. Данные способы сведения в дальнейшем будут уточнены. Для проблем полноты подобных способов сведения неизвестно. Это является еще одним аргументом в пользу изучения вместо проблемы полноты более общей проблемы выразимости.

Проблема выразимости возникает при изучении систем дискретных функций, когда $\mathcal{M}$ есть упорядоченная включением решетка функциональных подмножеств, в которой замкнутость понимается как замкнутость относительно некоторого заданного набора операций суперпозиции, а неравенство $Y \subseteq X^{\prime}$ означает, что каждая функция множества $Y$ выражается через функции множества $X$ посредством операции суперпозиции. Данная проблема имеет смысл и в более общей ситуации, когда $\mathcal{M}$ есть упорядоченная включением решетка всех подмножеств некоторой алгебры, всевозможные подалгебры которой составляют множество замкнутых элементов решетки, а неравенство $Y \subseteq X^{\prime}$ означает, что все элементы множества $Y$ выражаются термами, в которых функциональные символы интерпретируются как основные операции алгебры, а предметные переменные принимают значения в множестве $X$.

\section{2. Нижние окрестности}

Естественным средством решения проблемы выразимости элемента $Y$ полной решетки $\mathcal{M}$ с замыканием ' является указание некоторой системы $\mathscr{S}$ ее замкнутых элементов такой, что для любого элемента $X$ из $\mathcal{M}$ выполнение включения $X^{\prime} \supseteq Y$ равносильно отсутствию в $\mathscr{Y}$ элемента $Z$, включающего $X$. Всякая такая система $\mathscr{S}$ будет называться далее нижней окрестностью элемента $Y$ в решетке $\mathcal{M}$ с замыканием '. Иначе, нижняя окрестность элемента $Y$ в полной решетке $M$ с замыканием ' - это всякая такая система $\mathscr{Y}$ ее замкнутых элементов, не включающих $Y$, что всякий не включающий $Y$ замкнутый элемент $X$ решетки $\mathcal{M}$ включен в некоторый элемент $Z$ из $\mathscr{Y}$. Введенное понятие нижней окрестности обобщает понятие критериальной системы (см., например, [4]), используемое при решении проблемы полноты. Нижняя окрестность называется безызбыточной, если она состоит из попарно несравнимых по отношению включения элементов. Всякий элемент $Y$ решетки с замыканием имеет в ней нижнюю окрестность, например, тривиальную, состоящую из всех замкнутых элементов решетки, не включающих $Y$. Вместе с тем, некоторые элементы решетки могут не иметь безызбыточной нижней окрестности.

Отметим, что всякая нижняя окрестность элемента $Y$ в полной решетке $\mathcal{M}$ с замыканием включает систему $\mathscr{S}(Y)$ всех ее $Y$-максимальных элементов, а безызбыточная нижняя окрестность элемента $Y$ (если она существует) совпадает с системой $\mathscr{S}(Y)$. В частности, если система $\mathscr{S}(Y)$ является нижней окрестностью элемента $Y$, то эта система является наименьшей по включению и единственной безызбыточной нижней окрестностью элемента $Y$. Из конечной нижней окрестности элемента $Y$ (опять в случае ее существования) всегда можно выделить подсистему, являющуюся его безызбыточной нижней окрестностью. В связи с этим, если элемент $Y$ обладает хотя бы одной конечной нижней окрестностью, то система $\mathscr{S}(Y)$ является нижней окрестностью этого элемента, причем конечной, безызбыточной и наименьшей по включению. Легко понять также, что в решетке с замыканием $\mathscr{B}(P)$ замкнутый класс, имеющий конечную нижнюю окрестность, конечно порожден.

Как уже отмечалось, в ряде случаев проблема выразимости элемента решетки допускает сведение к формально более простым проблемам выразимости. Уточним этот тезис. Имеется по крайней мере две возможности. Во-первых, в решетке $\mathcal{M}$ с замыканием объ- 
единение $B_{\mathscr{A}}=\bigcup_{A \in \mathscr{A}} B_{A}$ нижних окрестностей $B_{A}$ элементов $A$ системы $\mathscr{A} \subseteq M$ является нижней окрестностью объединения $\bigcup \mathscr{A}$ этой системы. При этом нижняя окрестность $B_{\mathscr{A}}$ может не быть безызбыточной, даже если все нижние окрестности $B_{A}$ безызбыточные. Тем не менее, отмеченное свойство сводит проблему выразимости объединения к ряду проблем выразимости объединяемых элементов. Во-вторых, для любых элементов $A$ и $B$ решетки $\mathcal{M}$ с замыканием объединение $\mathscr{S}=\mathscr{S}_{1} \cup \mathscr{S}_{2}$ нижних окрестностей $\mathscr{S}_{1}$ и $\mathscr{S}_{2}$ элементов $A$ и $A \cup B$ в соответствующих решетках $\mathcal{M}$ и $\mathcal{M}_{A}$ является нижней окрестностью объединения $A \cup B$ в решетке $\mathcal{M}$. Здесь также нижняя окрестность $\mathscr{S}$ может оказаться избыточной, несмотря на безызбыточность нижних окрестностей $\mathscr{Y}_{1}$ и $\mathscr{S}_{2}$. Но все же проблема выразимости объединения $A \cup B$ в решетке $M$ сводится к проблемам выразимости элементов $A$ и $A \cup B$ в соответствующих решетках $M$ и $M_{A}$ с индуцированными порядками и замыканиями.

Представляют интерес условия, при которых заданный элемент полной решетки с замыканием имеет в ней конечную нижнюю окрестность или имеет в ней безызбыточную нижнюю окрестность. Проблема поиска таких условий обобщает проблемы, поставленные и частично решенные в [4]. В частности, представляют интерес условия, при которых произвольный или заданный конечно порожденный замкнутый класс решетки $\mathscr{B}(P)$ с замыканием имеет в ней конечную нижнюю окрестность. Эти условия дают обобщения некоторых известных теорем дискретной математики таких, как теорема А. В. Кузнецова о полноте из [5, 6], а также теорема С. В. Яблонского о предикатно-описуемых классах из [7] (последней в силу соответствия Галуа из [8]). Упомянутые теоремы неоднократно обобщались и передоказывались, например, в связи с функциями $k$-значной логики в [9-11], в связи с дискретными функциями более общего вида в [12] и в более общей ситуации в [4]. Подобные обобщения не утратили своей актуальности, в частности, в связи с появлением (действительным и возможным) новых классов управляющих систем и в связи с необходимостью решать проблемы и выразимости для них. Подобные обобщения интересны также в связи с проблемой упрощения критериев полноты из [5, 6], о чем указывалось в [4].

В статье будут рассмотрены сначала довольно общие условия финитарности, являющиеся достаточными для существования у элемента полной решетки с замыканием безызбыточной нижней окрестности и являющиеся необходимыми для существования у него конечной нижней окрестности. Далее будет показана финитарность замыкания, заданного в решетке $\mathscr{B}(P)$ так, что все конечно порожденные замкнутые классы имеют конечные нижние окрестности. Затем будут установлены более конструктивные достаточные условия существования конечной нижней окрестности, допускающие в ряде случаев несложную проверку. Этим будет обобщена теорема А. В. Кузнецова о полноте. Наконец, в завершение будут установлены необходимые и достаточные условия существования конечной нижней окрестности у конечно порожденного элемента полной решетки $\mathscr{B}(P)$ с замыканием Галуа. Тем самым наряду с теоремой А. В. Кузнецова о полноте будет обобщена и теорема С. В. Яблонского о предикатно-описуемых классах. Попутно в статье будут рассмотрены возможности эффективного задания замкнутых элементов решетки с замыканием, в частности, элементов нижних окрестностей, конечными запрещающими множествами, а в случае замыкания Галуа и конечными описаниями.

\section{3. Слабо финитарные элементы}

В данном разделе будет рассмотрено условие слабой финитарности элемента полной решетки с замыканием, являющееся достаточным для существования у него безызбыточной 
нижней окрестности. Результаты данного раздела являются несложными обобщениями известных фактов из [2] и приводятся для полноты изложения и дальнейшего использования.

Систему $\mathscr{C}$ элементов решетки $\mathcal{M}$ называют цепью, если для любых элементов $A$ и $B$ из $\mathscr{b}$ верно, что $A \subseteq B$ или $B \subseteq A$. Для любого элемента $A$ решетки $\mathcal{M}$ систему $\mathscr{C}$ (возможно не являющуюся цепью) будем называть $A$-мажорирующей, если $A$-мажорирующим элементом является ее объединение $\bigcup \mathscr{C}$, то есть если выполняется неравенство $A \subseteq(\bigcup \mathscr{C})^{\prime}$. Из введенных определений следует, что для любого элемента $A$ полной решетки $M$ с замыканием равносильны следующие условия:

(1) всякая $A$-мажорирующая цепь содержит $A$-мажорирующий элемент;

(2) всякая $A$-мажорирующая цепь замкнутых элементов содержит $A$-мажорирующий элемент.

Элемент $A$, обладающий свойствами (1) и (2), будем называть слабо-финитарным элементом решетки с замыканием $\mathcal{M}$. В соответствии с определением, слабая финитарность элемента $A$ равносильна слабой финитарности его замыкания $A^{\prime}$. В качестве примера отметим, что в финитарной решетке конечные множества, а также конечно порожденные замкнутые классы обладают свойством слабой финитарности. Без названия это свойство для наибольшего элемента решетки с замыканием в связи с проблемами конечной порождаемости и полноты замкнутых классов функций $k$-значной логики рассматривалось в [6]. Данное свойство представляет интерес в связи с проблемами выразимости, поскольку справедливо следующее утверждение.

Теорема 1. В полной решетке $М$ с замыканием для любого слабо финитарного элемента А система $\mathscr{Y}(A)$ является нижней окрестностью элемента $A$.

Справедливость данной теоремы следует из теоремы Куратовского-Цорна, см. [1], условиям которой в силу слабой финитарности элемента $A$ удовлетворяет упорядоченная отношением включения система всех замкнутых элементов из $\mathcal{M} \backslash \mu_{A}$.

Поскольку, как отмечалось, в полной решетке с замыканием объединение нижних окрестностей системы элементов является нижней окрестностью объединения этой системы, справедливо следующее утверждение.

Следствие 1. Пусть $\mathscr{A}$ - некоторая система слабо финитарных элементов решетки $\mathcal{M}$ с замыканием'. Тогда система $\bigcup_{A \in \mathscr{A}} \mathscr{S}(A)$ является нижней окрестностью объединения $\bigcup \mathscr{A}$.

Итак, теорема 1 дает достаточные условия существования у элемента полной решетки с замыканием безызбыточной нижней окрестности. Из этой теоремы, в частности, следует, что конечно порожденные замкнутые элементы финитарной полной решетки $\mathscr{B}(P)$ с замыканием обладают безызбыточными нижними окрестностями. Следствие 1 сводит проблему выразимости объединения $\bigcup \mathscr{A}$ системы $\mathscr{A}$ финитарных элементов полной решетки к нахождению $A$-максимальных элементов для $A \in \mathscr{A}$. В частности, в финитарной решетке $\mathscr{B}(P)$ проблема выразимости множества $A$ сводится к нахождению $\{a\}$-максимальных множеств для всевозможных элементов $a$ из $A$.

\section{4. Финитарные элементы}

В данном разделе будет введено в рассмотрение условие финитарности элемента полной решетки с замыканием, являющейся необходимым для существования у него конечной 
нижней окрестности.

Систему $\mathscr{C}$ элементов решетки $\mathcal{M}$ называют направленной вверх, если в системе $\mathscr{C}$ для любых ее элементов $A$ и $B$ найдется элемент, включающий объединение $A \cup B$.

Лемма 1. В полной решетке с замыканием для любого элемента А следуюшие условия равносильны:

(1) всякая А-мажсрирующая система содержит конечную А-мажсрирующую подсистему;

(2) всякая A-мажорирующая направленная вверх система содержит A-мажорирующий элемент;

(3) всякая A-мажорирующая направленная вверх система замкнутых элементов содержит А-мажорирующий элемент.

В полной решетке $\mathcal{M}=\mathscr{B}(P)$ с замыканием каждое из условий (1)-(3) равносильно условию

(4) всякое A-мажорирующее множество B из $\mathscr{B}(P)$ имеет конечное А-мажорирующее подмножество.

Доказательство. Пусть имеет место первое условие и $\mathscr{A}$ - направленная вверх $A$-мажорирующая система элементов рассматриваемой решетки. Тогда в силу первого условия в $\mathscr{A}$ можно выделить конечную $A$-мажорирующую подсистему $\mathscr{B}$. В силу конечности системы $\mathscr{B}$ и направленности вверх системы $\mathscr{A}$ в последней имеется элемент $C$, включающий объединение $\bigcup \mathscr{B}$. В силу транзитивности элемент $C$ является $A$-мажорирующим. Таким образом, имеет место второе условие, из которого третье условие следует очевидным образом. Пусть имеет место третье условие и $\mathscr{A}$ - некоторая $A$-мажорирующая система. Рассмотрим систему $\mathscr{B}$, состоящую из замыканий всевозможных конечных объединений элементов из $\mathscr{A}$. Так как $\mathscr{B}$ - направленная вверх и $A$-мажорирующая система, по третьему условию некоторый ее элемент $B$ является $A$-мажорирующим. Но элемент $B$ является замыканием объединения некоторой конечной системы элементов из $\mathcal{A}$, которая, очевидно, является $A$-мажорирующей. Таким образом, имеет место первое условие и доказана равносильность условий (1)-(3). Покажем теперь равносильность условий (2) и (4) в решетке $M=\mathscr{B}(P)$. С этой целью рассмотрим в ней $A$-мажорирующий элемент $B$ и отметим, что система всех конечных подмножеств множества $B$ является $A$-мажорирующей и направленной вверх. Вследствие этого второе условие влечет четвертое. Обратно, если $\mathscr{A}$ - некоторая $A$-мажорирующая направленная вверх система элементов решетки $\mathscr{B}(P)$, то в соответствии с определением элемент $\bigcup \mathscr{A}$ также является $A$-мажорирующим. Если имеет место четвертое условие, то найдется конечное $A$-мажорирующее подмножество $B \subseteq \bigcup \mathscr{A}$. В силу конечности множества $B$ и направленности вверх системы $\mathscr{A}$ в ней найдется множество $C$, включающее $B$. Такое $C$ является $A$-мажорирующим. Таким образом, из четвертого условия следует второе. Лемма доказана.

Всякий элемент $A$, удовлетворяющий условиям леммы 1 , будем называть финитарным. Как видно, финитарность элемента $A$ равносильна финитарности его замыкания $A^{\prime}$. В качестве примера отметим, что конечно порожденные замкнутые элементы финитарной решетки финитарны (выше отмечалась слабая финитарность таких элементов) и, как будет видно в дальнейшем, отмеченное свойство является характеризующим для финитарной решетки. Несложно показать, что финитарные замкнутые элементы решетки 
$\mathscr{B}(P)$ с замыканием конечно порождены, делать этого мы не будем. Из сказанного следует, что в финитарной (и только финитарной) решетке $\mathscr{B}(P)$ для замкнутого элемента условия конечной порожденности и финитарности равносильны.

Свойство финитарности формально сильнее свойства слабой финитарности. Тем не менее, в некоторых случаях эти свойства равносильны. Некоторые такие случаи будут рассмотрены далее.

Теорема 2. В полной решетке с замыканием для элемента А такого, что система $\mathscr{S}(A)$ конечна, следующие условия равносильны:

(1) элемент А финитарен;

(2) элемент А слабо финитарен;

(3) элемент А имеет конечную нижнюю окрестность.

Доказательство. Из первого условия второе следует очевидным образом, а из второго третье следует по теореме 1. Докажем, что из третьего условия следует первое. Пусть имеет место третье условие теоремы и $\mathscr{A}-A$-мажорирующая направленная вверх система элементов полной решетки. В силу третьего условия система $\mathscr{Y}(A)$ является конечной нижней окрестностью элемента $A$. Вследствие этого для любого элемента $B$ из $\mathscr{T}(A)$ в системе $\mathscr{A}$ найдется не включенный в него элемент $A_{B}$. Далее, в силу конечности системы $\mathscr{S}(A)$ и верхней направленности системы $\mathscr{A}$, в последней можно выбрать элемент $C$, включающий все элементы $A_{B}$. Такой элемент $C$ не включен ни в один из элементов критериальной системы $\mathscr{S}(A)$, и следовательно, является $A$-мажорирующим. Этим доказано, что элемент $A-$ финитарный. Таким образом, имеет место первое условие. Теорема доказана.

Непосредственно из нее получаем следующее утверждение.

Следствие 2. В полной решетке с замыканием для любого элемента А следующие условия равносильны:

(1) элемент А финитарен и система $\mathscr{S}(A)$ конечна;

(2) элемент А слабо финитарен и система $\mathscr{S}($ A) конечна;

(3) элемент А имеет конечную нижнюю окрестность.

Еще один случай, когда понятия финитарного и слабо финитарного элементов совпадают, дает следующая теорема.

Теорема 3. Пусть $P$ - счетное множество. Тогда для любого элемента А полной решетки $\mathscr{B}(P)$ с замыканием следующие условия равносильны:

(1) элемент А финитарен;

(2) элемент А слабо финитарен.

Доказательство. Отметим, что в решетке $\mathscr{B}(P)$ с замыканием $A$-мажорируемость ее элемента $B=\left\{b_{1}, b_{2}, \ldots\right\}$ равносильна $A$-мажорируемости цепи, составленной из элементов $\left\{b_{1}\right\},\left\{b_{1}, b_{2}\right\}, \ldots$ При этом слабая финитарность элемента $B$ означает, что некоторый элемент $\left\{b_{1}, \ldots, b_{n}\right\}$ этой цепи является $A$-мажорирующим. Таким образом, в $A$-мажорирующем множестве $B$ удается найти конечное $A$-мажорирующее подмножество. Тем самым в силу условия (4) леммы 1 и определения финитарности показано, что из второго условия следует первое. Поскольку обратная импликация очевидна, теорема доказана. 


\section{5. Слабо финитарные и финитарные замыкания}

В этом разделе будут рассмотрены замыкания, заданные в решетке $\mathscr{B}(P)$ так, что все ее конечно порожденные замкнутые элементы финитарны или слабо финитарны. Будет установлена (уже упоминавшаяся) финитарность замыкания в полной решетке $\mathscr{B}(P)$, при котором все ее конечно порожденные замкнутые элементы обладают конечными нижними окрестностями.

Лемма 2. Для полной решетки $\mathscr{B}(P)$ с замыканием следующчие условия равносильны:

(1) каждое конечное множество А из $\mathscr{B}(P)$ слабо финитарно;

(2) каждое одноэлементное множество $\{a\}$ из $\mathscr{B}(P)$ слабо финитарно;

(3) объединение любой цุепи замкнутых элементов замкнуто.

Доказательство. Очевидно, что из первого условия следует второе. Пусть выполняется второе условие. Рассмотрим произвольную цепь $\mathscr{C}$ замкнутых элементов из $\mathscr{B}(P)$. Очевидно, что выполняется включение $\bigcup \mathscr{C} \subseteq(\bigcup \mathscr{C})^{\prime}$. С другой стороны, для любого элемента $p$ из $(\bigcup \mathscr{C})^{\prime}$ цепь $\mathscr{b}$ является $\{p\}$-мажорирующей. В силу второго условия, в цепи $\mathscr{C}$ есть $\{p\}$-мажорирующий элемент $B$. Для него выполняются включения $\{p\} \subseteq B^{\prime}=B \subseteq \bigcup \mathscr{C}$. Следовательно, элемент $p$ принадлежит объединению $\mathscr{\ell}$. Таким образом, имеет место равенство $\bigcup \mathscr{C}=(\bigcup \mathscr{C})^{\prime}$ и из второго условия следует третье. Пусть теперь имеет место третье условие и $A-$ конечное множество из $\mathscr{B}(P)$. Пусть также $\mathscr{C}-A$-мажорирующая цепь замкнутых элементов из $\mathscr{B}(P)$. Тогда, используя третье условие, получаем равенство $A \subseteq(\bigcup \mathscr{C})^{\prime}=\bigcup \mathscr{C}$. Отсюда, в силу конечности множества $A$, следует, что цепь $\mathscr{b}$ имеет $A$-мажорирующий элемент. Таким образом, элемент $A$ слабо финитарен. Первое условие выполняется. Лемма доказана.

Замыкания в решетке $\mathscr{B}(P)$, обладающие свойствами (1)-(3) из леммы 2 , будем называть слабо финитарными. Аналогично лемме 2 доказывается следующее утверждение.

Лемма 3. Для полной решетки $\mathscr{B}(P)$ с замыканием следующчие условия равносильны:

(1) каждое конечное множество А из $\mathscr{B}(P)$ финитарно;

(2) каждое одноэлементное множество $\{a\}$ из $\mathscr{B}(P)$ финитарно;

(3) объединение любой направленной вверх системы замкнутых элементов замкнуто.

Мы опускаем доказательство этой леммы. Его легко получить из доказательства леммы 2, если в последнем вместо цепей $\mathscr{b}$ рассматривать направленные вверх системы.

В [3] показано, что условие (3) (а значит и каждое из условий (1) и (2)) равносильно условию финитарности замыкания, заданного в решетке $\mathscr{B}(P)$. Иными словами, условия леммы 3 равносильны возможности так определить на множестве $P$ структуру универсальной алгебры, чтобы всевозможные ее подалгебры составляли систему замкнутых элементов решетки $\mathscr{B}(P)$. Таким образом, каждое из условий леммы 3 дает еще одно определение финитарного замыкания.

Следствие 3. В полной решетке $\mathscr{B}(P)$ подмножеств счетного множества $P$ замыкание тогда и только тогда финитарно, когда оно слабо финитарно.

Данное следствие непосредственно получается из теоремы 3. Следующее утверждение вытекает из теоремы 2. 
Следствие 4. Для полной решетки $\mathscr{(}(P)$ с замыканием следующие условия равносильны:

(1) каждый конечно порожденный замкнутый элемент имеет конечную нижнюю окрестность;

(2) замыкание финитарно (равносильным образом слабо финитарно) и для каждого конечного (равносильно одноэлементного) множества А из $\mathscr{B}(P)$ система $\mathscr{S}(A)$ конечна.

В условии (2) следствия 4 свойство финитарности можно заменить свойством слабой финитарности и независимо от этого свойство конечности можно заменить свойством одноэлементности (на это указывается в скобках). Иными словами, условие (2) дает четыре равносильных свойства. Обратим внимание на следующий полученный выше результат: если в полной решетке $\mathscr{B}(P)$ с замыканием все конечно порожденные элементы имеют конечные нижние окрестности, то замыкание финитарно. Легко привести пример, указывающий на то, что обратное утверждение не имеет места. В качестве такого примера можно взять бесконечную циклическую группу с замыканием в решетке ее подмножеств относительно ее групповых операций.

\section{6. Предупорядочения и запрещающие множества}

В дальнейшем нас будут интересовать достаточные условия, при которых конечно порожденные замкнутые элементы финитарной решетки имеют в ней конечные нижние окрестности. В данном разделе будут даны некоторые определения, нужные для того, чтобы сформулировать такие условия. Пусть в множестве $P$ задано (рефлексивное и транзитивное) отношение предпорядка $\leqslant$. Наследственным классом предупорядоченного множества $(P, \leqslant)$ будем называть всякое подмножество $B \subseteq P$, которое вместе с любым своим элементом $b$ содержит и всякий элемент $a$ из $P$, удовлетворяющий неравенству $a \leqslant b$. Для множества $A \subseteq P$ через $A \leqslant$ будет обозначаться наименьший по включению класс среди наследственных классов предупорядоченного множества $(P, \leqslant)$, включающих множество $A$. Этот класс состоит из всех элементов $b$ из $P$ таких, что для некоторого элемента $a$ из $A$ имеет место неравенство $b \leqslant a$. Понятно, что можно говорить также о наследственных классах $A \leqslant$ множества $P$, предупорядоченного отношением $\geqslant$, двойственным к отношению $\leqslant$. Ясно, что дополнения наследственных классов предупорядоченного множества $(P, \leqslant)$ являются наследственными в $(P, \geqslant)$ и наоборот. Следовательно, всякий наследственный класс $B$ предупорядоченного множества $(P, \leqslant)$ можно задать указанием некоторого такого множества $C \subseteq P$, что $P \backslash B=C \geqslant$. Такое множество $C$ будем называть запрещающим для наследственного класса $B$ в предупорядоченном множестве $(P, \leqslant)$. Далее, предупорядочение $\leqslant$ будем называть согласованным с замыканием ', заданным в полной решетке $\mathscr{B}(P)$, если всякий замкнутый элемент этой решетки является наследственным классом предупорядоченного множества $(P, \leqslant)$, то есть, если для любого множества $A$ из $\mathscr{B}(P)$ имеет место равенство

$$
A_{\leqslant}^{\prime}=A^{\prime} .
$$

В этом случае замкнутые элементы решетки $\mathscr{B}(P)$ можно задавать посредством запрещающих множеств, как это было описано выше. Часто такой способ задания оказывается эффективным. Примером согласованного с замыканием ', заданным в решетке $\mathscr{B}(P)$, предупорядочения множества $P$ является предупорядочение $\leqslant$, определенное как

$$
x \leqslant y \Longleftrightarrow\{x\}^{\prime} \subseteq\{y\}^{\prime}
$$


для любых $x$ и $y$ из $P$. Это предупорядочение будем называть индуцированным в множестве $P$ замыканием '.

Замечание 1. Следует отметить, что наследственные классы комбинаторных объектов, таких, как дискретные функции, графы и др., естественным образом возникают в различных областях дискретной математики, таких, как теория графов, теория функциональных систем, математическая логика и др., и являются в настоящее время одним из классических объектов ее изучения. Так, наследственными классами при подходящем определении предупорядочения являются наследственные множества графов из [3], замкнутые операциями взятия миноров, инвариантные классы булевых функций из [14], замкнутые операциями перестановки переменных, введения и удаления фиктивных переменных и операциями подстановки констант, а также рассматриваемые в [7, 12] наследственные множества дискретных функций, замкнутые операциями перестановки и отожествлением переменных, а также операциями введения и удаления фиктивных переменных. Частным случаем наследственных классов дискретных функций являются замкнутые классы функций $k$-значной логики. Задание наследственных классов посредством запрещающих множеств оказывается часто важной и нетривиальной задачей. Классическим примером тому служит теорема Куратовского-Вагнера из [13], которая в терминах запретов описывает (наследственное) множество планарных графов. Другой пример дает лемма о немонотонной функции, вместе с другими леммами, участвующими в доказательстве теоремы Поста о полноте систем булевых функций [7]. Важность данной задачи обусловлена тем, что задание наследственных классов конечными запрещающими множествами в ряде случаев, например, во всех упомянутых выше случаях, позволяет получить алгоритм распознавания принадлежности этим классам. Некоторые условия, при которых замкнутые классы функций $k$-значной логики, рассматриваемые как наследственные множества, могут быть заданы посредством конечного множества запретов, установлены в [7]. В [12] подобные условия сформулированы для наследственных систем дискретных функций.

\section{7. Обобщение теоремы А. В. Кузнецова для решеток с замыканием}

Ближайшая цель данной статьи - найти достаточные условия того, что финитарный замкнутый класс решетки $\mathscr{B}(P)$ имеет конечную нижнюю окрестность, в которой элементы задаются конечными множествами запретов при некотором согласованном с замыканием предупорядочении множества $P$. Справедливо следующее утверждение.

Лемма 4. Пусть предупорядочение $\leqslant$ множества $P$ согласовано с замыканием', заданнылм в полной решетке $\mathscr{B}(P)$. Пусть также $B_{1}$ и $B_{2}-$ такие элементы решетки $\mathscr{B}(P)$, что для любого элемента $C$ из $\mathscr{B}(P)$ имеет место включение

$$
C^{\prime} \cap B_{1} \subseteq\left(C_{\leqslant} \cap B_{2}\right)^{\prime}
$$

Тогда в решетке $\mathscr{B}(P)$ с замыканием' для любого замкнутого класса $Q$ такого, что $\left(Q \cap B_{1}\right)^{\prime}=Q$, и любого элемента $Q_{1}$ из системь $\mathscr{S}(Q)$ имеет место равенство

$$
P \backslash Q_{1}=\left(B_{2} \backslash Q_{1}\right)_{\geqslant}
$$

Иньли словами, множество $B_{2} \backslash Q_{1}$ является запрещающим для наследственного класса $Q_{1}$ в предупорядоченном множестве $(P, \leqslant)$. Если элемент $Q$ финитарен в решетке 
$\mathscr{B}(P)$ с замыканием' и множество $B_{2}$ конечно, то элемент $Q$ имеет в решетке $\mathscr{B}(P)$ с замыканием' конечную нижнюю окрестность $\mathscr{S}(Q)$, для каждого элемента которой $в$ предупорядоченном множестве $(P, \leqslant)$ имеется конечное запрещуающее множество.

Доказательство. Включение

$$
P \backslash Q_{1} \supseteq\left(B_{2} \backslash Q_{1}\right) \geqslant
$$

следует из согласованности предпорядка $\leqslant$ с замыканием ' ${ }^{\prime}$. Докажем обратное включение. С этой целью выберем произвольно элемент $h$ из множества $P \backslash Q_{1}$. Предположим, что этот элемент не принадлежит множеству $\left(B_{2} \backslash Q_{1}\right) \geqslant$. В этом случае множества $\{h\} \leqslant$ и $\left(B_{2} \backslash Q_{1}\right)$ не пересекаются, и следовательно, имеет место включение

$$
\{h\} \leqslant \cap B_{2} \subseteq Q_{1} \cap B_{2} .
$$

Воспользовавшись этим включением и условиями леммы, получаем следующую последовательность включений:

$$
\begin{aligned}
Q \cap B_{1} & \subseteq\left(Q_{1} \cup\{h\}\right)^{\prime} \cap B_{1} \subseteq\left(\left(Q_{1} \cup\{h\}\right)_{\leqslant} \cap B_{2}\right)^{\prime} \\
& =\left(\left(Q_{1 \leqslant} \cup\left(\{h\}_{\leqslant}\right)\right) \cap B_{2}\right)^{\prime}=\left(\left(Q_{1} \cup\left(\{h\}_{\leqslant}\right)\right) \cap B_{2}\right)^{\prime} \\
& =\left(\left(Q_{1} \cap B_{2}\right) \cup\left(\{h\}_{\leqslant} B_{2}\right)\right)^{\prime}=\left(Q_{1} \cap B_{2}\right)^{\prime} \subseteq Q_{1} .
\end{aligned}
$$

Отсюда следует, что

$$
Q=\left(Q \cap B_{1}\right)^{\prime} \subseteq Q_{1}^{\prime}=Q_{1}
$$

Полученное противоречие доказывает требуемое включение, а с ним и равенство

$$
P \backslash Q_{1}=\left(B_{2} \backslash Q_{1}\right) \geqslant
$$

Из этого равенства в случае конечного множества $B_{2}$ следует конечность системы $\mathscr{T}(Q)$, которая по теореме 2 при финитарном элементе $Q$ является его нижней окрестностью. Лемма доказана.

В связи с доказанной леммой введем следующее определение. Предупорядочение $\leqslant$ множества $P$, согласованное с замыканием ', заданным в решетке $\mathscr{B}(P)$, будем называть сильно согласованным с этим замыканием, если найдется покрывающая множество $P$ направленная вверх система $\mathcal{N}$ конечных множеств из решетки $\mathscr{S}(P)$ и функция $\alpha: \mathcal{N} \rightarrow \mathcal{N}$ такая, что для любых множеств $B_{1} \in \mathcal{N}, B_{2}=\alpha\left(B_{1}\right)$ и $C \in \mathscr{B}(P)$ выполняется включение (1) из леммы 4. В этой ситуации будем говорить также, что предупорядочение $\leqslant$ сильно согласовано с замыканием' при помощи пары $(\mathcal{N}, \alpha)$. Следствием леммы 4 является следующее утверждение.

Теорема 4. Пусть предупорядочение $\leqslant$ множества $P$ сильно согласовано с финитарным замыканием', заданным в решетке $\mathscr{B}(P)$. Тогда в этой решетке всякий конечно порожденный замкнутый элемент имеет конечную нижнюю окрестность, для каждого элемента которой в предупорядоченном множестве $(P, \leqslant)$ имеется конечное запрещающее множество.

Доказательство. Пусть в условиях теоремы предупорядочение согласовано с замыканием при помощи пары $(\mathcal{N}, \alpha)$ и $Q$ есть замкнутый элемент решетки $\mathscr{B}(p)$, имеющий конечное порождающее множество $A$ такое, что $Q=A^{\prime}$. Выберем в системе $\mathcal{N}$ элемент 
$B_{1}$ такой, что $A \subseteq B_{1}$. Это можно сделать, поскольку множество $A$ конечное, система $\mathcal{N}$ направлена вверх, и ее объединение совпадает с $P$. Тогда условия леммы 4 выполняются для выбранного выше множества $B_{2}=\alpha\left(B_{1}\right)$. Для завершения доказательства осталось заметить, что $Q=\left(Q \cap B_{1}\right)^{\prime}$ и применить лемму 4. Теорема доказана.

Приведем пару примеров использования этой теоремы.

Пример 1. Пусть $E$ - конечное $k$-элементное множество. Через $P_{E}$ принято обозначать множество всевозможных операций $f: E^{n} \rightarrow E$ при натуральных $n=0,1, \ldots$, а через $P_{E}^{(n)}$ принято обозначать множество всех функций из $P_{E}$, зависящих не более, чем от $n$ переменных. В полной решетке $\mathscr{B}\left(P_{E}\right)$ будем считать определенной операцию замыкания относительно суперпозиции (см. $[5,6,9,10])$. Замыкание множества $A$ функций из $P_{E}$ относительно суперпозиции принято обозначать через $[A]$. Множество $[A]$ состоит из всевозможных функций, вычисляемых (реализуемых) схемами (равносильным образом формулами) над $A$; определение схемы можно найти в [15]. На множестве $P_{E}$ определим предупорядочение $\leqslant$, считая $f \leqslant g$, если функцию $f$ можно получить из функции $g$ в результате перестановки и отождествления переменных. Очевидно, что такое предупорядочение согласовано с замыканием относительно суперпозиции.

Лемма 5. Для любого множества $C$ функций из $P_{E}$ и любых натуральных $n$ и $N$ таких, что $N=k^{k^{n}}$ имеет место включение

$$
[C] \cap P_{E}^{(n)} \subseteq\left[C_{\leqslant} \cap P_{E}^{(N)}\right] .
$$

Доказательство. Выберем произвольно функцию $f$ из пересечения $[C] \cap P_{E}^{(n)}$. Иными словами, функция $f$ зависит не более, чем от $n$ переменных, например, от переменных $x_{1}, \ldots, x_{n}$, и вычисляется некоторой схемой над $C$, то есть такой схемой, в которой элементы вычисляют некоторые функции из $C$ (см. [15]). Из этой схемы, удаляя некоторые ее элементы и отождествляя входные полюсы ее элементов, можно построить схему над множеством $C_{\leqslant}$, в которой на различных внутренних вершинах (под внутренней вершиной схемы будем понимать вход или выход любого ее элемента, отличный от выхода схемы) вычисляются различные функции и которая по-прежнему вычисляет функцию $f$. Функции, вычисляемые на вершинах рассматриваемых схем (исходной и преобразованной), зависят от переменных $x_{1}, \ldots, x_{n}$. Этих функций не более, чем $k^{k^{n}}$. Поскольку в преобразованной схеме на различных внутренних вершинах вычисляются различные функции, число ее внутренних вершин не превосходит числа $N=k^{k^{n}}$. Тем более не превосходит этого числа число входов любого элемента преобразованной схемы. Таким образом, элементы преобразованной схемы реализуют функции, зависящие не более, чем от $N=k^{k^{n}}$ переменных и принадлежащие множеству $C_{\leqslant} \cap P_{E}^{(N)}$. Это означает, что функция $f$ принадлежит множеству $\left[C_{<} \cap P_{E}^{(N)}\right]$. Лемма доказана.

Иными словами, лемма 5 утверждает, что предупорядочение $\leqslant$ множества $P_{E}$ сильно согласовано с замыканием относительно суперпозиции при помощи пары $(\mathcal{N}, \alpha)$, где направленная вверх система $\mathcal{N}$ состоит из всевозможных множеств $P_{E}^{(n)}$ и $\alpha\left(P_{E}^{(n)}\right)=P_{E}^{(N)}$ для $N=k^{k^{n}}$. Используя теорему 4 , получаем теорему А. В. Кузнецова из $[5,6,9]$ в следующей обобщенной форме: всякий конечно порожденный замкнутый класс функций из $P_{E}$ имеет в решетке $\mathscr{B}\left(P_{E}\right)$ с замыканием относительно суперпозиции конечную нижнюю окрестность, для каждого элемента которой в предупорядоченном множестве $\left(P_{E}, \leqslant\right)$ имеется конечное запрещающее множество. 
Пример 2. Пусть теперь $E$ и $D-$ конечные множества. Будем обозначать через $P_{E, D}$ множество всевозможных функций $f: E^{n} \rightarrow D$ при всевозможных натуральных $n$ и чеpeз $P_{E, D}^{(n)}$ множество всевозможных функций из $P_{E, D}$, зависящих не более, чем от $n$ переменных. Пусть также заданы клоны (то есть содержащие тождественную функцию и замкнутые суперпозицией классы) $R$ и $L$ функций из $P_{E}$ и из $P_{D}$ соответственно. Множество $F$ функций из $P_{E, D}$ назовем $(L, R)$-замкнутым, если оно содержит суперпозицию (не обязательно бесповторную) $f(g, h, \ldots)$ всякий раз, когда $f \in F$ и $g, h, \ldots \in R$, а также всякий раз, когда $f \in L$ и $g, h, \ldots \in F$. Через $[C]_{L, R}$ будем обозначать наименьший по включению класс среди всех $(L, R)$-замкнутых классов, включающих $C$. Он состоит, как легко видеть, из всевозможных суперпозиций (не обязательно бесповторных) вида

$$
f=l\left(f_{1}\left(r_{11}, r_{12}, \ldots\right), f_{2}\left(r_{21}, f_{22}, \ldots,\right), \ldots\right),
$$

где функция $l$ принадлежит клону $L$, функции $f_{i}$ принадлежат множеству $C$, а функции $r_{i j}$ принадлежат клону $R$. Таким образом, в решетке $\mathscr{B}\left(P_{E, D}\right)$ оказывается определенной операция $(L, R)$-замыкания. Предупорядочение $\leqslant$ на множестве $P_{E, D}$ определим следующим образом: будем считать, что выполняется неравенство $f \leqslant g$, если функцию $f$ можно получить, отождествляя переменные функции $g$.

Лемма 6. Для любого множества $C$ функций из $P_{E, D}$ и любых натуральных $п$ и $N$ таких, что $N=\left|R \cap P_{E}^{(n)}\right|$ имеет место включение

$$
[C]_{L, R} \cap P_{E, D}^{(n)} \subseteq\left[C \leqslant \cap P_{E, D}^{(N)}\right]_{L, R}
$$

Доказательство. Выберем произвольно функцию $f$ из пересечения $[C]_{L, R} \cap P_{E, D}^{(n)}$. Иными словами, функция $f$ зависит не более, чем от $n$ переменных, и ее можно выразить суперпозицией вида (2). В этой суперпозиции функции $r_{i j}$ зависят не более, чем от $n$ переменных и принадлежат клону $R$. Среди этих функций различных не более $N$, поэтому для любого $i$ суперпозицию $f_{i}\left(r_{i 1}, r_{i 2}, \ldots\right)$ можно заменить равной ей функцией $f_{i}^{0}\left(r_{i 1}^{0}, r_{i 2}^{0}, \ldots\right)$, где каждая функция $f_{i}^{0}$ получается из функции $f_{i}$ отождествлением переменных (так, что $i$-я и $l$-я переменные отождествляются, если функции $r_{i j}$ и $r_{i l}$ совпадают) и при фиксированном $i$ все функции $r_{i j}^{0} \in R$ различны. Функции $f_{i}^{0}$ таким образом, зависят не более, чем от $N$ переменных и принадлежат множеству $C \leqslant \cap P_{E, D}^{(N)}$, а функция $f$ принадлежит множеству $\left[C_{\leqslant} \cap P_{E, D}^{(N)}\right]_{L, R}$. Это и требовалось доказать.

Иначе говоря, лемма 6 утверждает, что предупорядочение множества $P_{E, D}$ сильно согласовано с $(L, R)$-замыканием при помощи пары $(\mathcal{N}, \alpha)$, где $\mathcal{N}$ состоит из всевозможных множеств $P_{E, D}^{(n)}$ и $\alpha\left(P_{E, D}^{(n)}\right)=P_{E, D}^{(N)}$ для $N=\left|R \cap P_{E}^{(n)}\right|$. Используя теорему 4, получаем следующее обобщение теоремы А. В. Кузнецова: всякий конечно порожденный $(L, R)$-замкнутый класс функций из $P_{E, D}$ имеет в решетке $\mathscr{B}_{(}\left(P_{E, D}\right) c(L, R)$-замыканием конечную нижнюю окрестность, для каждого элемента которой в предупорядоченном множестве $\left(P_{E, D}, \leqslant\right)$ имеется конечное запрещающее множество.

Рассмотренные в данном примере операции $(L, R)$-замыкания вместе с $(L, R)$-замкнутыми классами функций из $P_{E, D}$ изучались в [2]. Такие $(L, R)$-замкнутые классы естественным образом возникают в теории управляющих систем как классы функций, реализуемых транзисторными или переключательными схемами с ограниченным или произвольным числом каскадов. 


\section{8. Проблемы полноты и выразимости в решетках с замыканием Галуа}

Рассмотрим теперь случай замыкания, индуцированного соответствием Галуа $[1,16]$ между решетками $\mathscr{B}(P)$ и $\mathscr{B}(T)$ подмножеств множества $P$ и множества $T$ соответственно. Пусть для каждого элемента $x$ из $P$ в множестве $T$ определено подмножество $x^{\alpha}$ и для каждого элемента $y$ из $T$ в множестве $P$ определено подмножество $y^{\beta}$. Для любых множеств $X$ из $\mathscr{B}(P)$ и $Y$ из $\mathscr{B}(T)$ положим

$$
X^{\alpha}=\bigcap_{x \in X} x^{\alpha}, \quad Y^{\beta}=\bigcap_{y \in Y} y^{\beta} .
$$

Тогда для любых подмножеств $X_{1}$ и $X_{2}$ множества $P$ и любых подмножеств $Y_{1}$ и $Y_{2}$ множества $T$ имеют место импликации

$$
\begin{gathered}
\left(X_{1} \subseteq X_{2}\right) \Longrightarrow\left(X_{2}^{\alpha} \supseteq X_{1}^{\alpha}\right), \\
\left(Y_{1} \subseteq Y_{2}\right) \Longrightarrow\left(Y_{2}^{\beta} \supseteq Y_{1}^{\beta}\right) .
\end{gathered}
$$

Потребуем также, чтобы для любых подмножеств $X \subseteq P$ и $Y \subseteq T$ выполнялись условия

$$
X \subseteq X^{\alpha \beta}, \quad Y \subseteq Y^{\beta \alpha} .
$$

В рассматриваемой ситуации говорят, что пара отображений $\alpha$ и $\beta$ таких, что

$$
\begin{array}{rlrl}
\alpha: \mathscr{B}(P) & \rightarrow \mathscr{B}(P), & \alpha: X \mapsto X^{\alpha}, \\
\beta: \mathscr{B}(T) \rightarrow \mathscr{B}(T), & \beta: Y \mapsto Y^{\beta}
\end{array}
$$

определяет соответствие Галуа между решетками $\mathscr{B}(P)$ и $\mathscr{B}(T)$. Хорошо известно (см. $[1,16])$, что в этом случае композиции

$$
\begin{array}{ll}
\alpha \beta: \mathscr{B}(P) \rightarrow \mathscr{B}(P), & \alpha \beta: X \mapsto X^{\alpha \beta}, \\
\beta \alpha: \mathscr{B}(T) \rightarrow \mathscr{B}(T), & \beta \alpha: Y \mapsto Y^{\beta \alpha}
\end{array}
$$

являются замыканиями в этих решетках. Данные замыкания принято называть замыканиями Галуа. Замкнутые ими элементы решеток $\mathscr{B}(P)$ и $\mathscr{B}(T)$ будем называть соответственно $\alpha \beta$-замкнутымим и $\beta \alpha$-замкнутыми, а также Галуа-замкнутыми. Из $[1,16]$ известно, что отображения $\alpha$ и $\beta$ индуцируют пару взаимно обратных инверсных изоморфизмов (см. [1]) между упорядоченными включением системами Галуа-замкнутых классов в решетках $\mathscr{B}(P)$ и $\mathscr{B}(T)$. Отметим, что для любого множества $A$ элементов из $P$ пара отображений $\alpha_{A}$ и $\beta$, где

$$
x^{\alpha_{a}}=(\{x\} \cup A)^{\alpha}=x^{\alpha} \cap A^{\alpha}
$$

для любого $x$ из $P$, определяет соответствие Галуа между решетками $\mathscr{B}(P)$ и $\mathscr{B}\left(A^{\alpha}\right)$. При этом $\alpha_{A} \beta$-замкнутыми классами в $\mathscr{B}(P)$ оказываются всевозможные $\alpha \beta$-замкнутые классы, включающие $A$, а $\beta \alpha_{A}$-замкнутые классы в $\mathscr{B}\left(A^{\alpha}\right)$ совпадают с $\beta \alpha$-замкнутыми. В связи с этим в решетках с замыканием Галуа проблема выразимости по-прежнему обобщает проблемы полноты, $A$-полноты и $A$-выразимости. 
Из сказанного ранее легко следует, что Галуа-замкнутыми элементами решетки $\mathscr{B}(P)$ являются всевозможные элементы $Y^{\beta}$, где $Y \in \mathscr{B}(T)$. Таким образом, всякий Галуазамкнутый класс $B$ решетки $\mathscr{B}(P)$ можно задать посредством указания некоторого такого множества $Y$ из $\mathscr{B}(T)$, что $B=Y^{\beta}$. Такое множество $Y$ будем называть описанием Галуа-замкнутого множества $B$ в множестве $T$. Аналогично, множество $X$ из $\mathscr{B}(P)$ такое, что $A=X^{\alpha}$, будем называть описанием Галуа-замкнутого множества $A$ в множестве $P$. Таким образом, в случае, когда замыкания в полных решетках индуцированы соответствием Галуа между ними, для задания замкнутых элементов наряду с известными ранее способами можно пользоваться описаниями. Часто задание посредством конечных описаний оказывается эффективным, в связи с чем вызывают интерес конечно-описуемые Галуа-замкнутые классы, обладающие конечными описаниями.

Далее будем считать, что в множествах $P$ и $T$ определены предупорядочения $\leqslant P$ и $\leqslant_{T}$, согласованные с замыканиями Галуа в соответствующих решетках $\mathscr{B}(P)$ и $\mathscr{B}(T)$. Это означает, что для любых элементов $x_{1}$ и $x_{2}$ из $P$ и любых элементов $y_{1}$ и $y_{2}$ из $T$ выполняются импликации

$$
\begin{aligned}
& \left(x_{1} \leqslant_{P} x_{2}\right) \Longrightarrow\left(x_{1}^{\alpha \beta} \subseteq x_{2}^{\alpha \beta}\right), \\
& \left(\begin{array}{ll}
y_{1} \leqslant T & y_{2}
\end{array}\right) \Longrightarrow\left(y_{1}^{\beta \alpha} \subseteq y_{2}^{\beta \alpha}\right)
\end{aligned}
$$

или, равносильным образом, выполняются импликации

$$
\begin{aligned}
& \left(\begin{array}{ll}
x_{1} \leqslant_{P} & x_{2}
\end{array}\right) \Longrightarrow\left(\begin{array}{ll}
x_{1}^{\alpha} \supseteq x_{2}^{\alpha}
\end{array}\right), \\
& \left(\begin{array}{ll}
y_{1} \leqslant T & y_{2}
\end{array}\right) \Longrightarrow\left(\begin{array}{ll}
y_{1}^{\beta} \supseteq y_{2}^{\beta}
\end{array}\right) .
\end{aligned}
$$

Частным случаем согласованных с замыканием Галуа предупорядочений являются предупорядочения, индуцированные замыканием Галуа, для которых приведенные выше импликации можно заменить равносильностями. Далее, наряду с проблемами полноты в решетке $\mathscr{B}(P)$ с замыканием Галуа, будем интересоваться условиями, при которых Галуа-замкнутые классы обладают конечными запрещающими множествами в предупорядоченных множествах $\left(P, \leqslant_{p}\right)$ и $\left(T, \leqslant_{T}\right)$.

Введем еще одно определение. В полной решетке $M$ с замыканием систему замкнутых элементов $\mathscr{H} \subseteq \mathcal{M}$ назовем верхней окрестностью замкнутого элемента $Y$, если для любого замкнутого элемента $X$ решетки $\mathcal{M}$ включение $X \subseteq Y$ равносильно отсутствию в $\mathscr{H}$ элемента $Z$, удовлетворяющего неравенству $Z \subseteq X$. Иначе говоря, элементы в $\mathscr{H}$ замкнуты, не включены в $Y$, и всякий не включенный в $Y$ замкнутый элемент решетки $\mathcal{M}$ включает некоторый элемент из $\mathcal{H}$. Отметим, что верхние окрестности, в отличие от нижних, определены только для замкнутых элементов решетки. Ясно, что инверсные изоморфизмы, установленные между системами замкнутых элементов полных решеток, переводят нижние окрестности замкнутых элементов на верхние окрестности образов этих элементов и обратно. В частности, существование конечной нижней окрестности элемента $X$ решетки $\mathscr{B}(P)$ равносильно существованию в решетке $\mathscr{B}(T)$ конечной верхней окрестности образа $X^{\alpha}$ этого элемента при отображении $\alpha$.

Теорема 5. Пусть отображения $\alpha: \mathscr{B}(P) \rightarrow \mathscr{B}(P)$ u $\beta: \mathscr{B}(T) \rightarrow \mathscr{B}(P)$ определяют соответствие Галуа между полными решетками $\mathscr{B}(P)$ и $\mathscr{B}(T)$. Если предупорядочение $\leqslant_{T}$ индуцировано в множестве $T$ замыканием Галуа, то для любых множеств $X$ из $\Re(P) u$ В из $\mathscr{B}(T)$ следующие условия равносильнь: 
(1) система

$$
\Lambda(B)=\left\{b^{\beta} \mid b \in B\right\}
$$

является нижней окрестностью множества $X$ в решетке $\mathscr{\Re}(P)$;

(2) множество В является запрещчающим для наследственного класса $X^{\alpha}$ в предупорядоченном множестве $\left(T, \leqslant_{T}\right)$, то есть

$$
T \backslash X^{\alpha}=B_{\geqslant} .
$$

Доказательство. Принадлежность элемента $y$ из $T$ множеству $x^{\alpha}$ равносильна условию $y^{\beta} \supseteq X^{\alpha \beta}$, и таким образом, равносильна тому, что класс $y^{\beta}$ является $X$-мажорирующим. Если имеет место условие (1), то свойство $X$-мажорируемости класса $y^{\beta}$ в свою очередь равносильно отсутствию класса $b^{\beta}$ в системе $\Lambda(B)$ (и элемента $b$ из $B$ ), обладающего свойством $y^{\beta} \subseteq b^{\beta}$, и таким образом, равносильно отсутствию элемента $b$ в $B$, удовлетворяющего неравенству $b \leqslant T y$; отсюда следует, что множество $B$ является запрещающим для наследственного класса $X^{\alpha}$ в предупорядоченном множестве $\left(T, \leqslant_{T}\right)$ и имеет место свойство (2).

Обратно, если выполняется свойство (2), то для любого множества $Z$ из $\mathscr{B}(P)$, не являющегося $X$-мажорирующим, в силу существования инверсного изоморфизма между системами замкнутых элементов решеток $\mathscr{B}(P)$ и $\mathscr{B}(T)$ множество $X^{\alpha}$ не является $Z^{\alpha}$-мажорирующим в решетке $\mathscr{B}(T)$, и следовательно, можно выбрать элемент $y$ в множестве $Z^{\alpha} \backslash X^{\alpha}$. В силу свойства (2) в множестве $B$ найдется элемент $b$, удовлетворяющий неравенству $b \leqslant_{T} y$. Тогда

$$
b^{\beta} \supseteq y^{\beta} \supseteq Z^{\alpha \beta} \supseteq Z
$$

и выполняется свойство (1). Теорема доказана.

Следствие 5. Пусть в условиях теоремы 5 предупорядочение $\leqslant_{T}$ множества $T$ согласовано с замыканием Галуа в полной решетке $\mathscr{B}(T)$. Тогда, если для множества $X$ из $\mathscr{B}(P)$ и В из $\mathscr{B}(T)$ имеет место равенство

$$
T \backslash X^{\alpha}=B_{\geqslant_{T}},
$$

то система $\Lambda(B)$ является нижней окрестностью элемента $X$ в решетке $\mathscr{B}(P)$ с замыканием Галуа.

Доказательство следует из теоремы 5, поскольку запрещающее множество Галуазамкнутого класса относительно любого согласованного с замыканием предупорядочения остается запрещающим и относительно предупорядочения, индуцированного замыканием.

Теорема 5 и следствие 5 сводят проблему выразимости множества $X$ в полной решетке $\mathscr{B}(P)$ с замыканием Галуа к нахождению в предупорядоченном множестве $(T, \leqslant T)$ запрещающего множества $B$ для наследственного класса $X^{\alpha}$. При этом возникает нижняя окрестность $\Lambda(B)$ множества $X$ в решетке $\mathscr{B}(P)$, состоящая из классов $b^{\alpha}$, где $b$ из $B$, имеющих конечные, более того, одноэлементные описания. Отметим, что такая нижняя окрестность $\Lambda(B)$ для множества $X$ обязательно существует, поскольку для наследственного класса $X^{\alpha}$ существует запрещающее множество $B$ (например, тривиальное $\left.B=T \backslash X^{\alpha}\right)$. Причем, если для $X$ существует безызбыточная (в частности, конечная) нижняя окрестность, то она может быть найдена как нижняя окрестность $\Lambda(B)$ для подходящего $B$. Далее более явно сформулируем условия существования конечных окрестностей у конечно порожденных замкнутых классов. 
Следствие 6. В условиях теоремы 5, для любого множества $X$ из $\mathscr{B}(P)$ следующие условия равносильнь:

(1) класс $X$ имеет в решетке $\mathscr{B}(P)$ с замыканием Галуа конечную нижнюю окрестность;

(2) существует согласованное с замыканием Галуа предупорядочение $\leqslant_{T}$ множества $T$ такое, что для наследственного класса $X^{\alpha}$ в предупорядоченном множестве $\left(T, \leqslant_{T}\right)$ имеется конечное запрещзаюее множество;

(3) если предупорядочение $\leqslant_{T}$ индуцировано в множестве $T$ замыканием Галуа, то для наследственного класса $X^{\alpha}$ в предупорядоченном множестве $\left(T, \leqslant_{T}\right)$ имеется конечное запрещзаюее множество.

Доказательство. Из первого условия третье следует в силу теоремы 5. Из третьего второе следует очевидным образом. Наконец, из второго условия первое получается по следствию 5. Следствие доказано.

Следствие 7. В условиях теоремы 5 следующие условия равносильны:

(1) в решетке $\mathscr{B}(P)$ с замыканием Галуа всякий конечно порожденный замкнутый класс имеет конечную нижнюю окрестность;

(2) существует согласованное с замыканием Галуа предупорядочение $\leqslant_{T}$ множества $T$ такое, что для любого элемента $х$ из $P$ для наследственного класса $x^{\alpha}$ в предупорядоченном множестве $\left(T, \leqslant_{T}\right)$ имеется конечное запрещуаючее множество;

(3) если предупорядочение $\leqslant_{T}$ индуцировано в множестве $T$ замыканием Галуа, то для любого элемента $x$ из $P$ для наследственного класса $x^{\alpha}$ в предупорядоченном множестве $\left(T, \leqslant_{T}\right)$ имеется конечное запрещуающее множество.

Следствие 7 доказывается аналогично предыдущему следствию с использованием следующего факта: условия существования конечных нижних окрестностей для всех одноэлементных множеств, для всех конечных множеств и для всех конечно порожденных замкнутых множеств равносильны.

Следствие 8. Пусть в условиях теоремы 5 предупорядочение $\leqslant_{T}$ множества $T$ согласовано с замыканием Галуа в решетке $\mathscr{B}(P)$, и для любого элемента х из $P$ наследственный класс $x^{\alpha}$ в предупорядоченном множестве $\left(T, \leqslant_{T}\right)$ имеет конечное запрещчаюшее множество. Тогда для любых Галуа-замкнутых классов $X$ из $\mathscr{B}(P)$ и $Y$ из $\mathscr{B}(T)$ таких, что $X^{\alpha}=Y$ (равносильным образом $X=Y^{\beta}$ ) следуюшие условия равносильнь::

(1) класс $X$ конечно порожден;

(2) класс $Y$ конечно описуем;

(3) класс $Y$ имеет в $\left(T, \leqslant_{T}\right)$ конечное запрещающее множество;

(4) класс $X$ имеет в решетке $\mathscr{B}(P)$ конечную нижнюю окрестность;

(5) класс $Y$ имеет в решетке $\mathscr{B}(T)$ конечную верхнюю окрестность. 
Доказательство. Ясно, что первые два и последние два условия между собой равносильны в силу существования инверсных изоморфизмов между системами замкнутых элементов решеток $\mathscr{B}(P)$ и $\mathscr{B}(T)$. Далее, из первого условия следует третье, так как, если Галуа-замкнутый класс $X$ имеет конечное порождающее множество, состоящее из элементов $x_{1}, \ldots, x_{n}$, то объединение конечных запрещающих множеств для классов $x_{1}^{\alpha}, \ldots, x_{n}^{\alpha}$ будет конечным запрещающим множеством для пересечения $x_{1}^{\alpha} \cap \ldots \cap x_{n}^{\alpha}$ этих классов, совпадающего, как можно видеть, с классом $Y$. В силу следствия 5 , третье условие влечет четвертое. Наконец, из четвертого очевидным образом следует первое. Следствие доказано.

Следствия 6 и 7 дают условия, при которых заданный или произвольный конечно порожденный замкнутый элемент решетки с замыканием Галуа имеет конечную нижнюю окрестность. Тем самым следствия 6 и 7 обобщают теорему А. В. Кузнецова о полноте. Следствие 8 наряду с теоремой А. В. Кузнецова о полноте обобщает теорему С. В. Яблонского о предикатно-описуемых классах.

Пример 3. Как в примере 1 , через $P_{E}$ будем обозначать множество всех операций на конечном $k$-элементном множестве $E$ и через $P_{E}^{(n)}$ будем обозначать множество всех не более чем $n$-арных операций из $P_{E}$. Условимся также обозначать через $\Pi_{E}$ множество всех предикатов на $E$ и через $\Pi_{E}^{(n)}$ обозначать множество не более чем $n$-арных предикатов на $E$. Для функции $f$ из $P_{E}$ через $\operatorname{inv}_{E}(f)$ обозначается множество всех предикатов из $\Pi_{E}$, сохраняемых функцией $f$; понятие сохраняемого предиката определено в $[7,8]$. Для предиката $p$ из $\Pi_{E}$ через $\operatorname{pol}_{E}(p)$ будем обозначать множество всех функций из $\Pi_{E}$, сохраняющих предикат $p$. Ясно, что пара отображений inv $E$ и $\operatorname{pol}_{E}$ определяет соответствие Галуа между решетками $\mathscr{B}\left(P_{E}\right)$ и $\mathscr{B}\left(\Pi_{E}\right)$. Из [8] известно, что в этом случае Галуа-замкнутыми классами функций являются всевозможные клоны (то есть содержащие тождественную функцию и замкнутые суперпозицией классы) функций из $P_{E}$, а Галуа-замкнутыми классами предикатов являются всевозможные множества предикатов, содержащие отношение равенства и замкнутые операциями проектирования, конъюнкции, отождествления и перестановки переменных. Определим предупорядочение $\leqslant$ в каждом из множеств $P_{E}$ и $\Pi_{E}$ так, что неравенство $f \leqslant g$ означает, что функция (предикат) $f$ получена из функции (предиката) $g$ путем отождествления и перестановки переменных. Несложно видеть, что данные предупорядочения согласованы с замыканиями Галуа. Отметим, что из любого предиката, не сохраняемого $n$-местной функцией, путем отождествления переменных можно получить предикат арности не более, чем $k^{n}$, не сохраняемый той же функцией, и из любой функции, не сохраняющей $n$-арный предикат, которому удовлетворяют $m$ наборов, где $m \leqslant k^{n}$, путем отождествления переменных можно получить функцию, зависящую не более чем от $m$ переменных и также не сохраняющую этот предикат. В связи с этим справедливо следующее утверждение.

Лемма 7. Для натуральных $n$ и $N=k^{n}$ функиии $f$ из $P_{E}^{(n)}$ и предиката р из $\Pi_{E}^{(n)}$ имеют место равенства

$$
\begin{aligned}
\Pi_{E} \backslash \operatorname{inv}_{E}(f) & =\left(\Pi_{E}^{(N)} \backslash \operatorname{inv}_{E}(f)\right)_{\geqslant}, \\
P_{E} \backslash \operatorname{pol}_{E}(p) & =\left(P_{E}^{(N)} \backslash \operatorname{pol}_{E}(p)\right)_{\geqslant}
\end{aligned}
$$

Иными словами, лемма 7 говорит о том, что наследственный класс $\operatorname{inv}_{E}(f)$ имеет в предупорядоченном множестве $\left(\Pi_{E}, \leqslant\right)$ конечное запрещающее множество $\Pi_{E}^{(N)} \backslash \operatorname{inv}_{E}(f)$ и наследственный класс $\operatorname{pol}_{E}(p)$ имеет в предупорядоченном множестве $\left(P_{E}, \leqslant\right)$ конечное запрещающее множество $P_{E}^{(N)} \backslash \operatorname{pol}_{E}(p)$. 
Лемма 7 позволяет воспользоваться доказанными ранее утверждениями в случае, когда множества $P$ и $T$ совпадают с соответствующими множествами $P_{E}$ и $\Pi_{E}$, а отображения $\alpha$ и $\beta$ совпадают с соответствующими отображениями $\operatorname{inv}_{E}$ и $\operatorname{pol}_{E}$. Таким путем из следствия 8 получается обобщенная теорема А. В. Кузнецова в следующей форме: клон функций из $P_{E}$ тогда и только тогда конечно порожден, когда он имеет конечную нижнюю окрестность в решетке $\Re_{(}\left(P_{E}\right)$ с замыканием относительно суперпозиции.

Также лемма 7 позволяет применить следствие 8 в случае, когда множества $P$ и $T$ совпадают с множествами $\Pi_{E}$ и $P_{E}$ соответственно, а отображения $\alpha$ и $\beta$ совпадают

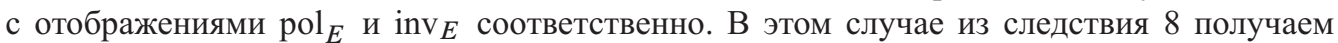
теорему Яблонского из [7] о предикатно-описуемых классах в следующей форме: клон $Q$ функиий из $P_{E}$ тогда и только тогда имеет конечное описание $B \subseteq \Pi_{E}$ такое, что $Q=\operatorname{pol}_{E}(B)$, когда клон $Q$ имеет конечную верхнюю окрестность в решетке $\mathscr{B}_{(}\left(P_{E}\right) c$ замыканием Галуа.

Пример 4. По-прежнему, как в примере 2, для заданных конечных множеств $E$ и $D$ через $P_{E, D}$ будем обозначать множество всех функций $f: E^{n} \rightarrow D$ при всевозможных натуральных $n$. Пару $(a, b) m$-арных предикатов $a$ из $\Pi_{E}$ и $b$ из $\Pi_{D}$ будем называть $m$-арным 2-предикатом (на паре множеств $E$ и $D$ ). Множество всех таких $m$-арных 2 -предикатов при всевозможных натуральных $m$ будем обозначать через $\Pi_{E, D}$. Введем понятие 2-предиката, сохраняемого функцией $f$ из $P_{E, D}$. С этой целью для $n$-местной функции $f$ из $P_{E, D}$ и натурального $m$ определим $n$-местную функцию $f^{[m]}$ из $P_{E^{m}, D^{m}}$, положив

$$
f^{[m]}\left(x_{1}, \ldots, x_{n}\right)=\left(f\left(x^{1}\right), \ldots, f\left(x^{m}\right)\right)
$$

для наборов $x_{i}=\left(x_{i 1}, \ldots, x_{i m}\right)$ из $E^{m}$ и наборов $x^{j}=\left(x_{1 j}, \ldots, x_{n j}\right)$ из $E^{n}$ таких, что $1 \leqslant i \leqslant n$ и $1 \leqslant j \leqslant m$. Будем говорить, что функция $f$ сохраняет $m$-арный 2 -предикат $(a, b)$, если для любых наборов $x_{1}, \ldots, x_{n}$, удовлетворяющих предикату $a$, набор $f^{(m)}\left(x_{1}, \ldots, x_{n}\right)$ удовлетворяет предикату $b$. Обозначим через $\operatorname{inv}_{E, D}(f)$ множество всех 2 -предикатов из $\Pi_{E, D}$, сохраняемых функцией $f$ из $P_{E, D}$, а через $\operatorname{pol}_{E, D}(p)$ обозначим множество всех функций из $P_{E, D}$, сохраняющих 2-предикат $p$ из $\Pi_{E, D}$. Ясно, что пара отображений $\operatorname{pol}_{E, D}$ и inv $E, D$ определяет соответствие Галуа между решетками $\mathscr{B}_{(}\left(P_{E, D}\right)$ и $\mathscr{B}\left(\Pi_{E, D}\right)$. Желая рассматривать более общую ситуацию, предположим, что заданы клоны $R$ и $L$ функций из $P_{E}$ и $P_{D}$, соответственно, и положим

$$
\begin{aligned}
\Pi_{E, D}^{L, R} & =\Pi_{E, D} \cap\left(\operatorname{inv}_{E}(R) \times \operatorname{inv}_{D}(L)\right), \\
\operatorname{inv}_{E, D}^{L, R}(f) & =\Pi_{E, D}^{L, R} \cap \operatorname{inv}_{E, D}(f)
\end{aligned}
$$

для любой функции $f$ из $P_{E, D}$. Введенные множества $\Pi_{E, D}^{L, R}$ и $\operatorname{inv}_{E, D}^{L, R}(f)$ совпадают с соответствующими множествами $\Pi_{E, D}$ и $\operatorname{inv}_{E, D}(f)$, если клоны $R$ и $L$ состоят только из селекторных функций. Несложно понять, что пара отображений $\operatorname{pol}_{E, D}$ и inv $E, R$ oпределяет соответствие Галуа между решетками $\mathscr{B}\left(P_{E, D}\right)$ и $\mathscr{B}\left(\Pi_{E, D}^{L, R}\right)$. Данное соответствие Галуа изучалось в статье [12]. Несложно показать, и это было сделано в [12], что Галуазамкнутыми классами функций в этом случае являются всевозможные $(L, R)$-замкнутые классы функций из $P_{E, D}$ (определение которых содержится в примере 2). Также в [12] в явной форме были описаны Галуа-замкнутые множества 2-предикатов. Далее предупорядочения в множествах $P_{E, D}$ и $\Pi_{E, D}$ определим следующим образом. Для функций $f$ и $g$ из $P_{E, D}$ полагаем $f \leqslant g$, если, как в предыдущих примерах, функцию $f$ можно получить из функции $g$ путем отождествления и перестановки переменных. Будем считать, что для $n$-арного 2-предиката $p=(a, b)$ и $m$-арного 2-предиката $q=(c, d)$ из $\Pi_{E, D}$ выполняется 
неравенство $q \leqslant p$, если для некоторой функции $f:\{1, \ldots, n\} \rightarrow\{1, \ldots, m\}$ при любых значениях $x_{1}, \ldots, x_{n}$ из $E$ и любых значениях $y_{1}, \ldots, y_{n}$ из $D$ имеют место соотношения

$$
\begin{aligned}
& c\left(x_{1}, \ldots, x_{m}\right)=a\left(x_{f(1)}, \ldots, x_{f(n)}\right), \\
& d\left(y_{1}, \ldots, y_{m}\right)=b\left(y_{f(1)}, \ldots, y_{f(n)}\right) .
\end{aligned}
$$

Иными словами, предикаты $c$ и $d$ получаются в результате перестановки и отождествления переменных из соответствующих предикатов $a$ и $b$, причем отождествления и перестановки переменных в обоих случаях аналогичны. Непосредственно проверяется, что предупорядочения в множествах $P_{E, D}$ и $\Pi_{E, D}$ согласованы с замыканием Галуа. Рассуждая примерно так же, как в предыдущем примере, получаем следующую лемму 9.

Лемма 8. Для натуральных $n$ и $N=k^{n}$, функичии $f$ из $P_{E, D}^{(n)}$ и 2-предиката $p$ из $\Pi_{E, D}^{L, R(n)}$ имеют место равенства

$$
\begin{aligned}
\Pi_{E, D}^{L, R} \backslash \operatorname{inv}_{E, D}^{L, R}(f) & =\left(\Pi_{E, D}^{L, R(N)} \backslash \operatorname{inv}_{E, D}^{L, R}(f) \geqslant,\right. \\
P_{E, D} \backslash \operatorname{pol}_{E, D}(p) & =\left(P_{E, D}^{(N)} \backslash \operatorname{pol}_{E, D}(p)\right)_{\geqslant} .
\end{aligned}
$$

Иными словами, лемма 8 утверждает, что наследственный класс $\operatorname{inv}_{E, D}^{L, R}(f)$ имеет в предупорядоченном множестве $\left(\Pi_{E, D}^{L, R}, \leqslant\right)$ конечное запрещающее множество $\Pi_{E, D}^{L, R(T)} \backslash \operatorname{inv}_{E, D}^{L, R}(f)$ и наследственный класс $\operatorname{pol}_{E, D}(p)$ имеет в предупорядоченном множестве $\left(P_{E, D, \leqslant}\right)$ конечное запрещающее множество $P_{E, D}^{(N)} \backslash \operatorname{pol}_{E, D}(p)$.

Лемма 8 позволяет воспользоваться доказанными ранее утверждениями в случае, когда $P, T, \alpha$ и $\beta$ суть соответственно $P_{E, D}, \Pi_{E, D}, \operatorname{inv}_{E, D}^{L, R}$ и $\operatorname{pol}_{E, D}$. Тогда из следствия 8 получается следующее обобщение теоремы А. В. Кузнецова: $(L, R)$-замкнутый класс $Q$ функций из $P_{E, D}$ тогда и только тогда имеет конечное порождающее множество $A \subseteq P_{E, D}$ такое, что $Q=[A]_{L, R}$, когда класс $Q$ имеет конечную нижнюю окрестность в решетке $\mathscr{B}_{(}\left(P_{E, D}\right)$ с $(L, R)$-замыканием.

Также лемма 8 позволяет применить следствие 8 в случае, когда $P, T, \alpha$ и $\beta$ совпадают соответственно с $\Pi_{E, D}, P_{E, D}, \operatorname{pol}_{E, D}$ и $\operatorname{inv}_{E, D}^{L, R}$. Таким образом, получается следующее обобщение теоремы С. В. Яблонского: $(L, R)$-замкнутый класс $Q$ функиий из $P_{E, D}$ тогда и только тогда имеет конечное описание $B \subseteq \Pi_{E, D}^{L, R}$ такое, что $Q=\operatorname{pol}_{E, D}^{L, R}(B)$, когда класс $Q$ имеет конечную верхнюю окрестность в решетке $\mathscr{B}_{(}\left(P_{E, D}\right) c(L, R)$-замыканием.

\section{Список литературы}

1. Курош А. Г., Лекции по общей алгебре. Лань, Санкт-Петербург, 2005.

2. Кон П., Универсальная алгебра. Мир, Москва, 1968.

3. Birkhoff G., Frink O., Representations of lattices by sets. Trans. Amer. Math. Soc. (1948) 64, 299-316.

4. Кузнецов А. В., Структуры с замыканием и критерии функциональной полноты. Успехи матем. наук (1961) 16, №2, 201-202.

5. Яблонский С. В., Введение в дискретную математику. Наука, Москва, 1986.

6. Яблонский С. В., Функциональные построения в $k$-значной логике. Труды Математического института им. В. А. Стеклова АН СССР (1958) 51, 5-142. 
7. Яблонский С. В., О строении верхней окрестности для предикатно-описуемых классов в $P_{k}$. Доклады АН СССР (1974) 218, №2, 304-307.

8. Бондарчук В. Г., Калюжный Л. А., Котов В. Н., Ромов Б. А., Теория Галуа для алгебр Поста. Кибернетика (1969) №№3, 5, 1-10, 1-9.

9. Мальцев А. И., Итеративные алгебры Поста. Изд-во НГУ, Новосибирск, 1976.

10. Мальцев А. И., Итеративные алгебры и многообразия Поста. Алгебра и логика (1966) 5, №2, $5-24$.

11. Парватов Н. Г., Замечания о конечной порождаемости замкнутых классов. Дискретный анализ и исследование операщий, Сер. 1 (2004) 11, №3, 32-47.

12. Парватов Н. Г., Наследственные системы дискретных функций. Дискретный анализ и исследование операций, Сер. 2 (2007) 14, №2, 76-91.

13. Дистель Р., Теория графов. Изд-во Ин-та математики, Новосибирск, 2002.

14. Яблонский С. В., О невозможности элиминации перебора всех функций из $P_{2}$ при решении некоторых задач теории схем. Доклады АН СССР (1959) 124, №1, 44-47.

15. Лупанов О. Б., О синтезе некоторых классов управляющих систем. Проблемы кибернетики (1963) 10, 61-80.

16. Риге Ж., Бинарные отношения, замыкания, соответствия Галуа. Кибернетический сборник (1963) 7, 129-185.

Статья поступила 18.11.2008. 